\title{
Application research of functional training in the Athletic Training
}

\author{
Longfei Lin \\ Tianjin University of Sport \\ Tianjin,300381 China
}

\begin{abstract}
In this paper, literature review method is used, based on the functional fitness training, and in close connection with athletics projects point, having in-depth study of the basic content of athletics and physical training specific training methods. And this paper presented some physical training methods bear the characteristics of the project, combined with our physical problems to give some advice.
\end{abstract}

Keywords- Functional training, physical fitness, athletics

\section{INTRODUCTION}

Modern competitive sports competition are so brutal that athletes increased sports injuries results from increasing physical activity, in the rehabilitation process of sports injuries, some new theories and methods continue to be applied. Functional training, an important part of rehabilitation, has long been applied to the treatment of sports injuries among the country, and gradually is treated seriously after 2006, after the 2008 Olympics, functional strength training began carry on around the country. From rehabilitation medicine aspect, function refers to tissues, organs, limbs and other characteristics of activity, and functional training is targeted rehabilitation measures implemented in order to recover from tissues, organs, limbs dysfunction, and it is the core of modern rehabilitation, belonging important aspect of occupational therapy. Rehabilitation science, functional training is the core of rehabilitation and the basic therapy which aims to improve the treatment of people with disabilities. According to the anatomical and physiological features, functional training can be divided into neurological rehabilitation, cognitive rehabilitation, life functional rehabilitation training. Exercise function training is an important part of functional training rehabilitation, mainly in the development of muscle strength, body coordination and muscle proprioception-based training. In recent years, functional training has entered from the field of rehabilitation medicine to the sports training and athletic fields and are widely concerned and used. In sports and public health fields, functional training is about improving neuromuscular control, joint stability and muscle endurance based, including balance training, stability training and proprioceptive training. Pang Delong believes that functional training should be a broader concept, can be divided into functional strength, functional speed. Functional endurance is to find areas of individual athletes' weak points from relatively g lobal perspective, and functional strength training is one of the branches, tend to be a more general concept. The specific manifestations include balance training, core strength training, proprioceptive training, flexibility and sensitivity response training, vibration training and suspension training. Although functional strength training was merely a branch of functional training, but it is a very important one, and has played a very important value in competitive sports.

\section{THE CONCEPT OF FUNCTIONAL TRAINING}

The earliest use of functional fitness training is in the field of rehabilitation abroad, and after research and development is used in athletic training by foreign scholars, and achieved good results. Today in sports training in the United States and Europe, functional fitness training has occupied a very important position. Famous American physical education specialists Gray Cook, in 2003, proposed the concept of "best athletic ability pyramid", he thought if functional movement training is included into normal daily training of athletes, it would do a lot of good, such as improving the efficiency of the athletes; making training more efficient; and also plays a role in the prevention of injuries. Bill foran explains in a book: physical training at a high level athletics, functional train ing is training form of a realistic imitation of human body movement in the natural state, and is training methods by strengthening the core strength of the neuromuscular system to make training more efficient.

\section{FUNCTIONAL STRENGTH TRAINING IN ATHLETICS PROJECT APPLICATION}

The core area is the middle part of the formation of the body including waist, pelvis, hip, specifically the area below the shoulder above the hip, including the pelvis, which contains all the abdominal muscles with back, pelvic muscles and muscle constitute the pelvis. Athletics needs athletes to stop and begin quickly and running to complete the conversion action when remaining stagnant in the air, these actions need core power to control as a bas is to balance the body, in addition, to increase the quality of the game, an increase in training the core part of the main core strength 
training, that part of the training is mainly done by means of specialized systems, highlighting the core area of the power chain to complete shifting steps action role in the completion and final sprint. Comp lete high quality sprint action perfectly. This part of the training focused on the front part of the trunk muscle strength training, by instability, open chain action design, fully integrate sprint technical requirements, and increase contraction forces abdominals, external oblique, internal oblique, iliopsoas muscle in vacant state rectus to create the conditions for completion of the sprint action. Back pain is a major problem about athletics athlete injury, and waist is also the weakest link in human muscle, but plays an important role in maintaining body posture and technology transfer of power in action. Athletics waist twisting and landing buffering action, ask for high requirements of the waist muscles, including multi-column waist deep muscle, erector spine, gluteus and front and fascia. Lumbar back muscles are trained mainly with a single point, multi-point, multi-plane unstable open-chain action design, such as lower limb suspension stand, prone balance pad support and other action like that. Athletics have a lot of jerk action, the work of the buffering capacity have higher requirements of knee and ankle joints. Increase functional strength training in knee and ankle joints not only has an important significance for giving good performance, but also has a positive meaning to lower extremity injuries of the knee and ankle joints, the part of the training focused primarily on the overall play of knee-ankle motor function, and pay attention to the two joint associated muscles, ligaments and nerve-based training, focusing on ankle proprioceptive training. In addition, specially designed upper body functional muscle training can enhance processing capability of field athletes in emergency situations of the game, such training mainly is body neuromuscular control training. Functional strength training's training goal is to improve the overall efficiency of the muscles and the ability to work and is also target training and the special training of sublimation, in functional strength training, it should be in conjunction with the technical characteristics of athletics, pay attention to muscular endurance and explosive of functional training, as well as handle traditional strength training, We should combine traditional strength training like electing the barbell and squats in barbell-room and functional strength training to solve basic training and strength training relationship, based on the theoretical basis of introduction, the traditional strength and functional strength training are made closer to the special ability. Wei Ankui pointed out; the greater difference of the form of strength training and the form of special activities, the smaller effect of their access to the special role to performance improvement. Apart from the form of functional strength training should close to the special activities, more important is the similarity in the chain work and special nerve. In the general strength training and functional strength training, the operation speed and exercise intensity is shown in the picture below, functional strength training using low-intensity resistance or conduct to overcome resistance and the traditional power adopt lager resistance intensity.

\section{FUNCTIONAL FIT NESS TRAINING METHODS OF ATHLETICS ATHLETES}

As to athletics athlete strength training, some trainer don't have a profound learning of the importance and role of the strength training, so it doesn't have a exact impact on the effects of strength training. American athletes do not develop muscles only from the track and field, but the results of specialized strength training. From the mechanical point of view, that if the human body wants to maximize strength and explosiveness, it must rely on the results of the maximum coordination between the various muscle movements, in order to run fast, jump high, one can not just practicing leg, distal peripheral joint development of explosive is a very important factor. In addition, trunk strength (waist and abdomen muscles and back muscles) is particularly important for athletics, but some athletes just noticed on the upper and lower extremity strength, while ignoring the area of body strength. For athletics both require explosive and power, two forces must be able to develop and rationally used in the training time and training methods arrangements should reflect a reasonable proportion of these two training, and it should be based explosive, fast power supplemented for strength training. According to the characteristics of athletics project and task in the competition period, force training methods should be effectively arranged, focusing on the development and activation of specific active muscle movement, muscle flexion amine under the amount of pressure on the knee and ankle muscles, trunk muscles, make an effort to play a role in the athletics competition. From the previous method of strength training of athletics athletes, it was found that lower limb squat exercised more, relatively few upper extremity training taken place, resulting in an imbalance of upper and lower extremity. Moreover, not enough emphasis on the standard action when athletes are having strength training, and not in accordance with the provisions of the actions required to complete, it will affect the results of training and likely to cause joint damage. Strength training arrangements contain training of the major muscle groups of the body in order to enhance the power level of athletics athletes. In practice the first thing is to regulate the operation mode, such as squats, deadlifts, bench press reps, etc., to slowly increase exercise load when achieving basic movements. After that, functional strength training are also arranged, such as the elastic band on the oblique twist and pull diagonally Downward, elastic contact with forward enough, and so before the kettle bell swing. As to the parts of the body which are easily to be hurt during track and field sports like knees and shoulders, each increase an enhanced resistance training, such as weight-bearing lunge position and sit three Swiss ball dumbbell. Finally, abdominal back muscles strength training, back muscles rotating under load exercise lower back power, sit-ups around the rod main training abdominal muscles.

At the same time also increased the ankle and other small muscle group training. Track and field athlete development focus. Speed training must be in combination with the biggest strength, speed, power and perfect mobile technology of action, at the same time also need technical movement to 
supplement, and the combination of special speed and specific skills The special speed training of track and field sport athletes mainly is about to increase the game starting skills and the ability to run quickly. Reaction speed training: speed training should be combined with special technology action of track and field. Main training methods are: to be familiar with special technology action, improve human perception, and shorten the response time of the each link. Movement speed training: the key of the action and speed training of track and field sport athletes is the key technical link. Main training methods: practice the key link of individual action and joint actions of composite moves, improve the speed of technical movement, raise the rate of complete technical movement, can let the track and field sport athletes complete the number or the maximu mumber of technical action within the prescribed time, to complete the action in the shortest time.

Move speed training: increase of displacement speed is greatly linked with and frequency of the action and movement range. The main training method is to improve the action speed and operating frequency. The improvement of frequency is taken place when under the circumstance of ensuring a certain movement range, make the action with as many as possible within the given time by improving the technology and improve the speed. The amplitude of the movement training is mainly achieved by improving the technology, improving muscle elasticity and fle xibility of the joints.

\section{ADVANT AGES OF FUNCTIONAL ST RENGTH TRAINING METHOD}

\section{A. Effectively increase weak muscle strength}

Athletes tend to cause sports damage because the power of the thigh muscle, foot muscle, tibialis anterior muscle too weak to have training, and the uneven developments of left and right leg power also affects the physical effect, while functional training can be selectively, accurately develop and strengthen a unit of muscle groups, and control muscle contraction strength and contraction time, then achieve a training effect which can not be brought by traditional methods, and in this way can the muscles get sufficient exercise. Therefore functional training is ideal for training methods to improve weak muscle strength.

\section{B. Able to develop fast-muscle force}

Functional strength training is a effective and safe exercise methods equal to great resistance training and isometric exercises, it is the only ideal means and methods of the current development of fast-twitch muscle fiber strength, because the human body mobilize slow twitch, and then fasttwitch muscle; while the e functional training is totally opposite, fast-twitch muscle mobilization followed by mobilization of slow muscle Therefore, strength training of speed and power project, should pay attention to the application of functional method.

\section{Is an ideal means of enhancing muscle strength}

Functional strength training muscle growth rate is significantly higher than traditional strength training, because functional training can maximize the mobilization of all units involved in the movement when the nerve center and the whole rest of the case are having rest, $(100 \%$ mobilized muscle fibers theoretically). Compared with the conventional method it has the advantages, and can be repeated, without the overall fatigue; sports center is not involved, so that no strong overall energy consumption. So overall recovery and adjustment capability are high, and is conducive to improve muscular strength.

\section{Injuries can shorten the rehabilitation process}

Functional training set strength training, health prevention, injury treatment at an organic whole, can guarantee the athlete to have muscle strength training and rehabilitation therapy simultaneously. Research has shown that compared with general recovery exercise, functional recovery exercise can get a better result, can relieve muscle atrophy, and will not reduce muscle metabolism, so as to shorten the recovery proces s, stable treatment effect.

\section{E. Athletes can improve relative muscle strength}

Functional training, as a strong external stimuli, and in the premise of mobilize fast-twitch fibers, can maximize the mobilization of muscle motor units involved in activities, and then can improve the coordination function of muscles, therefore, functional strength training will not result in muscle volume and cross-sectional growth when the power improved, and is a theoretical approach to improve relative muscle strength of athlete.

\section{REFERENCES}

[1] Liu JiaJin, Wang Qiang, Sun Zijian. Study on Grey System Theory in Athletic Training Application [J]. Chinese Sports Technology, 1999,11:25-27.

[2] Wang Lijuan, Zhou Bo. Chinese sports system reform two years [J]. SPORT S SCIENCE, 2002,06:4-6.

[3] Zhengai Lian. Electrical stimulation Strength Training in Track and Field Sports Training [J]. Kashgar Teachers College,2002,03:79-81.

[4] Zhang Dongli, Zhu Wen Bing. Comprehensive assessment of physical function application stat us and countermeasures in Athletic Training [J]. Capital institute of physical education,2001,04:85-87.

[5] State Planning Institute research group income gap between urban residents in China macroeconomic analysis moderation. [J]. Macroeconomic Research,2002,06:52-57.

[6] Liu Jiajin, Sunguiyun. GM $(1, \mathrm{~h})$ system state model [J] in track and field training. Shanghai Physical Education Institute,2003,01:78 -81 +95 .

[7] Conghua, Zhu Jinghua. Static exercises in Athletic Training Application [J]. Athletics,2004,09:54-55.

[8] Xiaolin Peng. Chinese Sports Management System Reform Research Review. [J]. Xi'an Institute of Physical,2005,01:23-26. 